\title{
Improvement of InGaN-GaN Light-Emitting Diode Performance With a Nano-Roughened p-GaN Surface
}

\author{
Hung-Wen Huang, C. C. Kao, J. T. Chu, H. C. Kuo, Member, IEEE, S. C. Wang, Member, IEEE, and C. C. Yu
}

\begin{abstract}
This investigation describes the development of InGaN-GaN light-emitting diode (LED) with a nano-roughened top p-GaN surface which uses $\mathrm{Ni}$ nano-mask and wet etching. The light output of the InGaN-GaN LED with a nano-roughened top p-GaN surface is 1.4 times that of a conventional LED, and wall-plug efficiency is $45 \%$ higher. The operating voltage of InGaN-GaN LED was reduced from 3.65 to $3.5 \mathrm{~V}$ at $20 \mathrm{~mA}$ and the series resistance was reduced by $20 \%$. The light output is increased by the nano-roughening of the top p-GaN surface. The reduction in the series resistance can be attributed to the increase in the contact area of nano-roughened surface.
\end{abstract}

Index Terms-Gallium nitride (GaN), light-emitting diode (LED), nano-mask, nickel.

$\mathbf{G}$ ALLIUM NITRIDE (GaN)-based materials have attracted considerable interest in relation to their potential use in optoelectronic devices, such as light-emitting diodes (LEDs) and laser diodes [1]. Recently, as the brightness of GaN-based LEDs has increased, applications such as displays, traffic signals, backlights for cell phones, exterior automotive lighting, and printers have become possible. However, the internal quantum efficiency of GaN-based LEDs is much less than $100 \%$ at room temperature because of nonradiative defects. Furthermore, the external quantum efficiency of GaN-based LEDs is low because the refractive index of the nitride epitaxial layer differ greatly from that of the air. The refractive indexes of $\mathrm{GaN}$ and air are 2.5 and 1.0, respectively. Thus, the critical angle at which light generated in the InGaN-GaN active region can escape is approximately $\left[\theta_{c}=\sin ^{-1}\left(n_{\text {air }} / n_{G a N}\right)\right] \sim 23^{\circ}$, which limits the external quantum efficiency of conventional GaN-based LEDs to only a few percent [2]. The light from LEDs can be enhanced either through the sample surface or through the side walls of the chip. Research into improving the light extraction efficiency (external quantum efficiency) and brightness in the LEDs [2]-[4] has been intense. Recently, Chang et al. reported that cap layers grown at low temperature $\left(800{ }^{\circ} \mathrm{C}\right)$ increased the power output by InGaN-GaN multiple quantum well (MQW) LEDs by $10 \%$ [5]. Fujii reported an increase in the extraction efficiency of GaN-based light-emitting diodes by surface roughening [6]. These processes all allow

Manuscript received September 9, 2004; revised December 6, 2004. This work was supported in part by the National Science Council of Republic of China (R.O.C.) in Taiwan under Contract NSC 92-2215-E-009-015, NSC 92-2112-M-009-026 and by the Academic Excellence Program of the R.O.C. Ministry of Education under the Contract 88-FA06-AB.

H.-W. Huang, C. C. Kao, J. T. Chu, H. C. Kuo, and S. C. Wang are with the Department of Photonics and Institute of Electro-Optical Engineering, National Chiao Tung University, Hsinchu 300, Taiwan, R.O.C., and also with TrueLight Corporation, Hsinchu 300, Taiwan, R.O.C. (e-mail: scwang@ @cc.nctu.edu.tw).

C. C. Yu is with the Global Union Technology Corporation, Hsinchu 300, Taiwan, R.O.C.

Digital Object Identifier 10.1109/LPT.2005.846741 the photons generated within the LEDs to find the escape cone by multiply scattering from a rough surface. Huh et al. reported that microroughening the top surface of an InGaN-GaN LED using metal clusters as a wet etching mask increased the wall-plug efficiency by $62 \%$ [2]. Huh et al. showed a large improvement in the light output power, indicating that the use of metal clusters to fabricate a roughened $\mathrm{p}-\mathrm{GaN}$ surface is an excellent means of making a high-power LED. However, higher treatment temperature of $900{ }^{\circ} \mathrm{C}$ is necessary to form Pt clusters. Therefore, higher temperature may increase the Indium segregation probability in the InGaN-GaN quantum well region and reduce the internal quantum efficiency [7], [8]. Pt has a high melting point and chemical stability [9]. Therefore, specific wet chemical etching should be necessary to remove Pt metal clusters by dipping into a boiling aqua-regia solution [2]. This investigation reports on the production of GaN LED with a nano-roughened $\mathrm{p}-\mathrm{GaN}$ surface using a self-assembled $\mathrm{Ni}$ metal cluster as the wet etching mask. The dimensions and density of the self-assembled Ni cluster can be controlled by rapid thermal annealing (RTA) at temperatures from $750{ }^{\circ} \mathrm{C}$ to $850^{\circ} \mathrm{C}$, details of which have been recently reported [10]. As a result, the light output efficiency of the LED with a nano-roughened surface was significantly higher than that of a conventional LED without a roughened surface. Additionally, the current-voltage $(I-V)$ measurements demonstrate that the forward voltage of an LED with a nano-roughened surface was lower than that of a conventional LED.

The GaN LED samples were grown by metal-organic chemical vapor deposition with a rotating-disk reactor (Emcore D75) on a $c$-axis sapphire (0001) substrate at a growth pressure of 200 mbar. Trimethylgallium, trimethylaluminum ammonia, $\mathrm{CP}_{2} \mathrm{Mg}$, and $\mathrm{Si}_{2} \mathrm{H}_{6}$ were used as sources of $\mathrm{Ga}, \mathrm{Al}, \mathrm{N}, \mathrm{Mg}$, and $\mathrm{Si}$. The LED structure includes a 30-nm-thick GaN low-temperature buffer layer, a $4.0-\mu \mathrm{m}$-thick highly conductive $\mathrm{Si}$-doped $\mathrm{GaN}$ layer (grown at $1050{ }^{\circ} \mathrm{C}$ ), an active region of undoped MQWs that includes $2 / 5$-nm-thick $\operatorname{In}_{0.21} \mathrm{Ga}_{0.79} \mathrm{~N}-\mathrm{GaN}$ with five periods of MQWs (grown at $750{ }^{\circ} \mathrm{C}$ ), a 50-nm-thick $\mathrm{Mg}$-doped $\mathrm{AlGaN}$ layer (grown at $1050{ }^{\circ} \mathrm{C}$ ), and finally $0.1-\mu \mathrm{m}$-thick Mg-doped $\mathrm{GaN}$ grown at $1050{ }^{\circ} \mathrm{C}$. The top surface of LED, which is a p-GaN surface, was roughened by both the formation of an Ni nano-mask on a top p-GaN surface of an LED and by wet etching. The surface roughness of the LED cap layer was measured by tapping mode atomic force microscopy (Veeco).

Nano-roughened LEDs were formed by depositing an Ni thin film with a thickness of $5 \mathrm{~nm}$ on a p-GaN surface by electron beam evaporation. RTA was then performed at $850^{\circ} \mathrm{C}$ for $1 \mathrm{~min}$ to change the Ni layer to the metal Ni nano-mask on the top p-GaN surface. Then, wet etching was performed to produce nano-roughened LEDs, using a boiling $85 \%$ phosphoric acid 
$\left(\mathrm{H}_{3} \mathrm{PO}_{4}\right)$ solution for 4-min etching. The etching rate of the p-GaN layer in boiling $85 \% \mathrm{H}_{3} \mathrm{PO}_{4}$ solution was determined to be approximately $15 \mathrm{~nm} / \mathrm{mim}$ at $200{ }^{\circ} \mathrm{C}$. The nano-roughened LED was dipped into a nitric acid solution $\left(\mathrm{HNO}_{3}\right)$ for $5 \mathrm{~min}$ to remove the Ni nano-mask from a nano-roughened LED after the wet etching process. Afterwards, the conventional LED and the LED with a nano-roughened surface were fabricated using the standard process (four mask steps) with a mesa area $\left(300 \times 300 \mu \mathrm{m}^{2}\right)$. First, the $0.5-\mu \mathrm{m} \mathrm{SiO}_{2}$ was deposited onto the sample surface by plasma-enhanced chemical vapor deposition. Photolithography was used to define the mesa pattern after wet etching of $\mathrm{SiO}_{2}$ by a buffer oxide etching solution. The mesa etching was then performed with $\mathrm{Cl}_{2}-\mathrm{Ar}$ as the etching gas in an inductively coupled plasma (ICP) reactive ion etching (ICP-RIE) system (SAMCO ICP-RIE 101iPH) which the ICP source power and bias power were operated at $13.56 \mathrm{MHz}$. The metal contact layers, including transparent contact and pad layers, were patterned by a liftoff procedure and deposited onto samples by electron beam evaporation. $\mathrm{Ni}-\mathrm{Au}(3 / 5 \mathrm{~nm})$ was used for the transparent electrode and $\mathrm{Ti}-\mathrm{Al}-\mathrm{Ni}-\mathrm{Au}$ $(20 / 150 / 20 / 200 \mathrm{~nm})$ was used for the n-type electrode. Finally, $\mathrm{Ni}-\mathrm{Au}(20 / 150 \mathrm{~nm})$ was deposited onto the p-type electrode.

Fig. 1(a)-(c) shows the atomic force microscopy (AFM) images that describe the change of the surface morphology of the $\mathrm{p}-\mathrm{GaN}$ surface during surface-roughening. Fig. 1(a) shows that the conventional $\mathrm{p}-\mathrm{GaN}$ cap has a root-mean-square (rms) roughness of $0.7 \mathrm{~nm}$, and a surface depth of approximately $2 \mathrm{~nm}$. The surface of the conventional LED was smooth. Fig. 1(b) shows a nano-mask AFM image rms roughness of $5.9 \mathrm{~nm}$ before wet etching was performed. The self-assembled Ni mask dimension size and density were approximately $250 \mathrm{~nm}$ and $2 \times 10^{9} \mathrm{~cm}^{-2}$, and the height of the Ni clusters was approximately $30 \mathrm{~nm}$ when the original $\mathrm{Ni}$ thickness was $50 \AA$ under RTA conditions of $850^{\circ} \mathrm{C}$ for $1 \mathrm{~min}$. Fig. 1(c) displays the AFM image that shows that rms roughness of $\mathrm{p}-\mathrm{GaN}$ surface increased drastically to $3.6 \mathrm{~nm}$, and the surface depth was approximately $15 \mathrm{~nm}$ after wet etching and the removal of the Ni nano-mask.

The $I-V$ characteristics of the conventional and nano-roughened LEDs were also measured. Fig. 2 plots the $I-V$ characteristics of conventional and nano-roughened LEDs. The forward voltages of the conventional and nano-roughened LEDs were 3.65 and $3.5 \mathrm{~V}$ at a driving current of $20 \mathrm{~mA}$, respectively. Furthermore, the dynamic resistance $(R=d V / d I)$ of the nano-roughened LED (32 $\Omega$ ) was $20 \%$ lower than that of the conventional LED $(40 \Omega$ ). The reduction in the series resistance of the LED with wet etching on a top nano-roughened LED surface can be attributed to the improvement in the ohmic contact resistance caused by the increase in the contact area [2]. The inset in Fig. 2 shows that the rough surface of the LED nano-roughened using an Ni nano-mask and wet etching did not induce a larger leakage current than that in the conventional LED. Furthermore, two devices, based on conventional and nano-roughened LEDs, in HBM (human body mode) were observed in an electrostatic discharge test at a reverse voltage of $>300 \mathrm{~V}$.

Electroluminescence (EL) was measured by injecting a continuous current into a device at room temperature. The light output was detected using a calibrated large-area Si photodiode
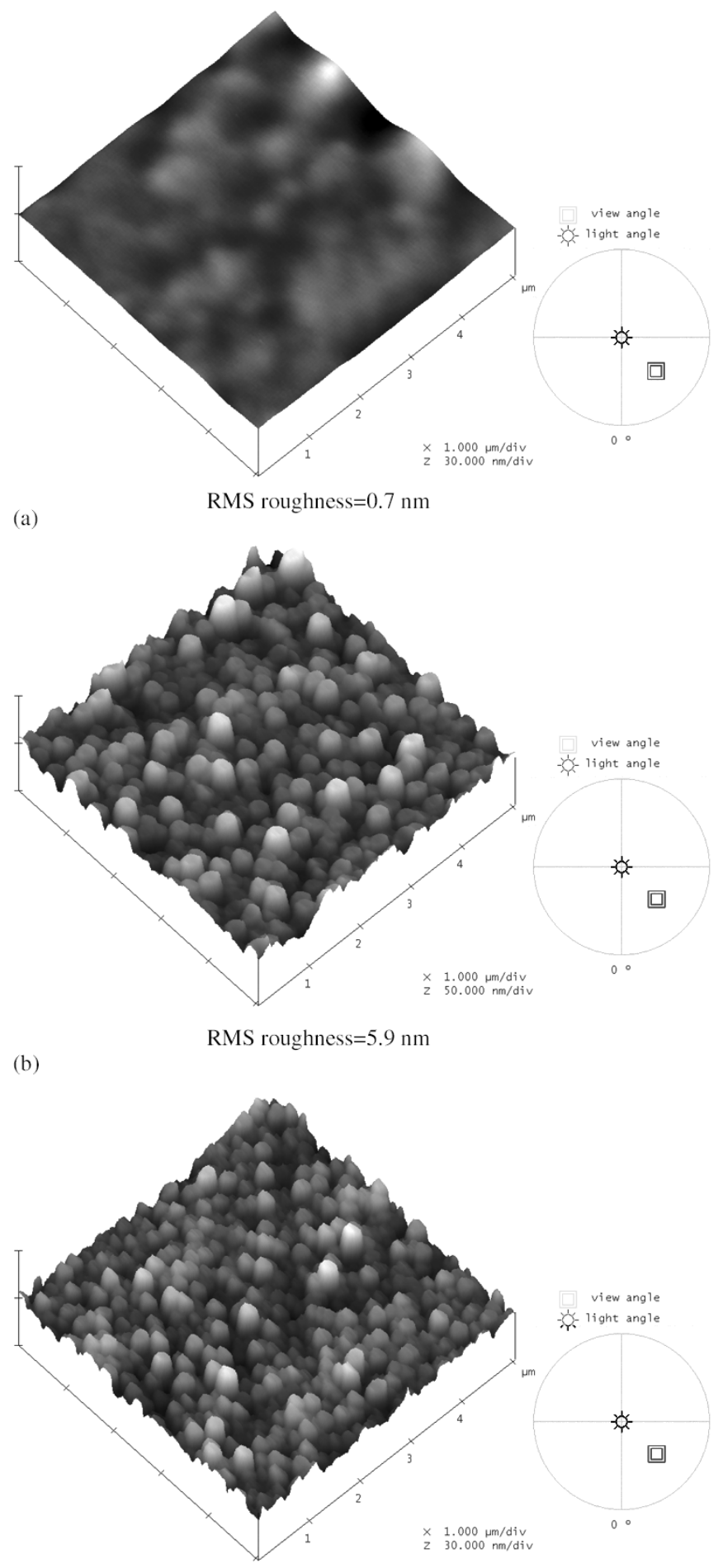

(c)

RMS roughness $=3.6 \mathrm{~nm}$

Fig. 1. AFM images of the top surface morphology of an LED sample. (a) Conventional LED p-GaN surface image. (b) Ni nano-mask on p-GaN surface image. (c) Nano-roughened LED top p-GaN surface image.

placed $5 \mathrm{~mm}$ from the top of the device. This detecting condition covers $\alpha$. Fig. 3(a) and (b) plots the spectra and intensity-current $(L-I)$ characteristics of conventional and nano-roughened LEDs. The EL intensity of the nano-roughened LED exceeds that observed from the conventional LED [as shown in Fig. 3(a)]. At an injection current of $20 \mathrm{~mA}$, all of the MQW emission peaks of these two were at approximately $450 \mathrm{~nm}$ and the 


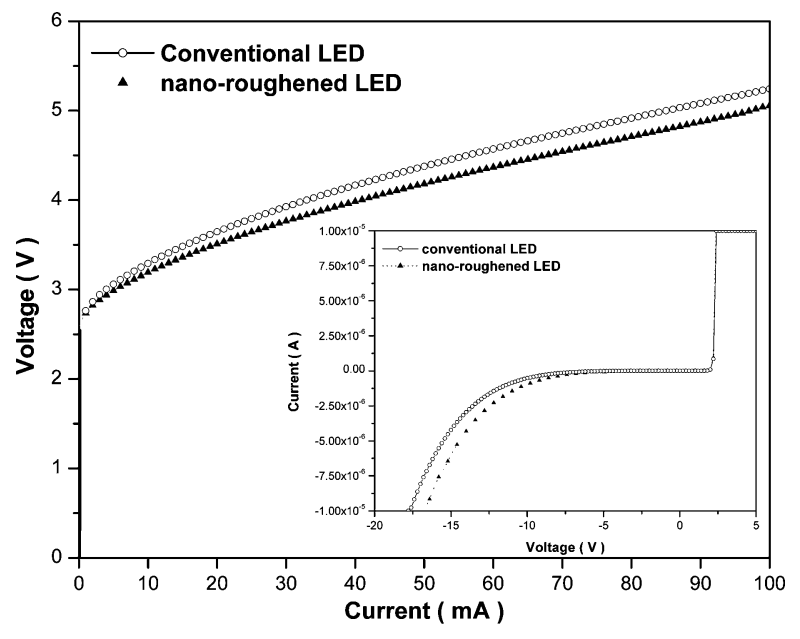

Fig. 2. $I-V$ forward curves of conventional and nano-roughened LEDs fabricated in this investigation. The inset shows reverse curves of the two devices.
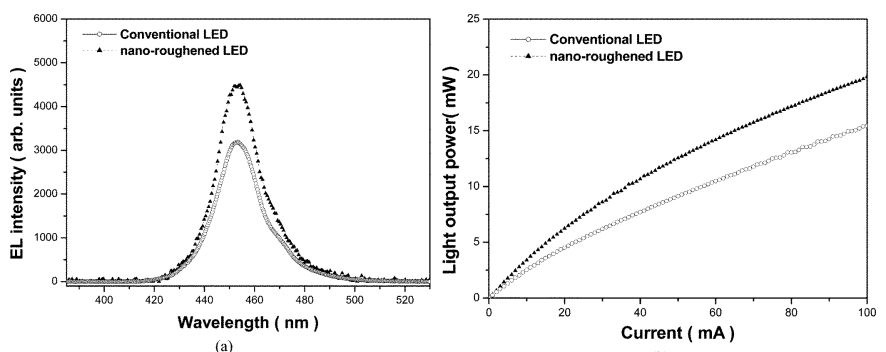

Fig. 3. (a) Room temperature EL spectrum of conventional and nano-roughened LED at a current of $20 \mathrm{~mA}$. (b) Light output power-current $(L-I)$ characteristics of conventional and nano-roughened LEDs.

light output power of the conventional and nano-roughened LEDs were approximately 4.5 and $6.3 \mathrm{~mW}$, respectively [as shown in Fig. 3(b)]. Restated, nano-roughening the p-GaN surface increased the output power of the InGaN-GaN MQW LEDs by a factor of 1.4 , indicating that the LED with the nano-roughened surface had larger light extraction efficiency. The wall-plug efficiency (output power/input power) was also calculated: It was $45 \%$ higher than that of the conventional LED at an injection current of $20 \mathrm{~mA}$, because of enhanced light output power and a lower forward voltage.

The intensity distributions of conventional and nano-roughened LEDs were measured to investigate further the influence of surface roughness on the light output performance of an LED. Fig. 4(a) and (b) shows the photons of conventional and nanoroughened LEDs when a $20-\mathrm{mA}$ dc current is injected into these two devices. Intensity distributions are also shown. The EL intensities observed from the nano-roughened LED clearly exceeded those from the conventional LED at the same injection current, especially on the LED top surface. Such an enhancement could be attributed to the top surface roughness and the fact that photons were more likely to be emitted from the surface-roughed device, resulting in an increase in the light output power of the nano-roughened LED, as shown in Fig. 3.

In summary, this investigation describes the improvement of an InGaN-GaN MQW LED by nano-roughening the $\mathrm{p}-\mathrm{GaN}$ surface using Ni nano-mask and wet etching. The nano-roughened surface improved the escape probability of photons

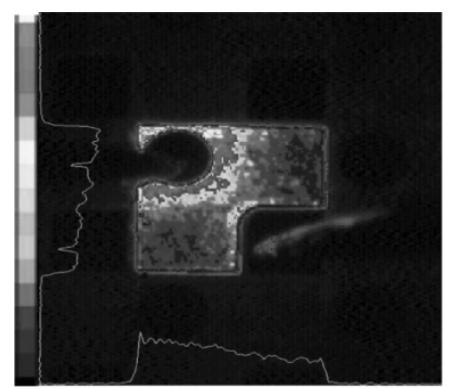

(a)

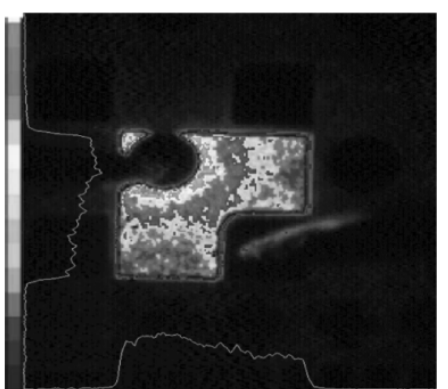

(b)
Fig. 4. Photons of (a) conventional LED and (b) nano-roughened LED at a dc injection current of $20 \mathrm{~mA}$.

inside the LED structure, increasing by $40 \%$ the light output of InGaN-GaN LED at $20 \mathrm{~mA}$. The operating voltage of the InGaN-GaN LED was reduced from 3.65 to $3.5 \mathrm{~V}$ at $20 \mathrm{~mA}$ and the series resistance was reduced by $20 \%$ by the increase in the contact area of the nano-roughened surface. The wall-plug efficiency of the InGaN-GaN LED was increased by $45 \%$ by nano-roughening the top $\mathrm{p}-\mathrm{GaN}$ surface using the Ni nano-mask and wet etching.

\section{ACKNOWLEDGMENT}

The authors would like to thank Prof. S. Wu, F. I. Lai, and T. H. Hseuh from National Chiao Tung University, Prof. J. K. Shen from National Chen Kung University, and Dr. C. F. Chu of Highlink Corporation for useful discussion.

\section{REFERENCES}

[1] S. Nakamura, M. Senoh, S. Nagahama, N. Iwasa, T. Yamada, T. Matsushita, Y. Sugimoto, and H. Kiyoku, "Room-temperature continuous-wave operation of InGaN multiquantum- well-structure laser diodes with a long lifetime," Appl. Phys. Lett., vol. 70, pp. 868-870, 1997.

[2] C. Huh, K. S. Lee, E. J. Kang, and S. J. Park, "Improved light-output and electrical performance of InGaN-based light-emitting diode by microroughening of the p-GaN surface," J. Appl. Phys., vol. 93, pp. 9383-9385, 2003.

[3] T. N. Oder, K. H. Kim, J. Y. Lin, and H. X. Jiang, "III-nitride blue and ultraviolet photonic crystal light emitting diodes," Appl. Phys. Lett., vol. 84, pp. 466-468, 2004.

[4] T. Fujii, Y. Gao, R. Sharma, E. L. Hu, S. P. DenBaars, and S. Nakamura, "Increase in the extraction efficiency of GaN-based light-emitting diodes via surface roughening," Appl. Phys. Lett., vol. 84, pp. 855-857, 2004.

[5] S. J. Chang, L. W. Wu, Y. K. Su, Y. P. Hsu, W. C. Lai, J. M. Tsai, J. K. Sheu, and C. T. Lee, "Nitride-Based LED's with $800{ }^{\circ} \mathrm{C}$ grown p-AlInGaN-GaN double-cap layers," IEEE Photon. Technol. Lett., vol. 16, no. 6, pp. 1447-1449, Jun. 2004.

[6] T. Fujii, Y. Gao, R. Sharma, E. L. Hu, S. P. DenBaars, and S. Nakamura, "Increase in the extraction efficiency of GaN-based light-emitting diodes via surface roughening," Appl. Phys. Lett., vol. 84, pp. 855-857, 2004.

[7] Y. S. Lin, K. J. Ma, C. C. Yang, and T. E. Weirich, "Effects of post-growth thermal annealing on the indium aggregated structures in InGaAN/GaN quantum wells," J. Cryst. Growth, vol. 242, pp. 35-40, 2002.

[8] C. J. Youn, T. S. Jeong, M. S. Han, J. W. Yang, K. Y. Lim, and H. W. Yu, "Influence of various activation temperatures on the optical degradation of Mg doped InGaN/GaN MQW blue LEDs," J. Cryst. Growth, vol. 250, pp. 1315-1322, 2003.

[9] H. Ishikawa, S. Kobayashi, Y. Koide, S. Yamasaki, S. Nagai, J. Umezaki, M. Koike, and M. Murakami, "Effects of surface treatments and metal work functions on electrical properties at p-GaN/metal interfaces," $J$. Appl. Phys., vol. 81, no. 3, pp. 1315-1322, 1997.

[10] H. W. Huang, C. C. Kao, T. H. Hsueh, C. C. Yu, C. F. Lin, J. T. Chu, H. C. Kuo, and S. C. Wang, "Fabrication of GaN-based nanorod light emitting diodes using self-assemble nickel nano-mask and inductively coupled plasma reactive Ion etching," Mater. Sci. Eng. B, vol. 113/2, pp. 125-129, 2004. 